\section{EARLY RESPIRATORY MANAGEMENT AND BRONCHOPULMONARY DYSPLASIA DEVELOPMENT IN VERY PRETERM NEWBORNS}

\author{
D. Dobryanskyy ${ }^{1}$, O. Borysiuk ${ }^{1,2}$, Z. Salabay ${ }^{2}$, \\ O. Novikova ${ }^{2}$
}

${ }^{1}$ Paediatrics, L'viv National Medical University, ${ }^{2}$ Neonatal Intensive Care Unit, L'viv Regional Clinical Hospital, L'viv, Ukraine

Bronchopulmonary dysplasia (BPD) remains important mostly unpreventable cause of morbidity of very preterm infants significantly affecting their long-term outcomes. In a cohort study we evaluated the possible protective effect of early noninvasive respiratory support on BPD development.

Methods: 100 very low birth weight newborns with gestational age of $28,37 \pm 2,0$ wk who survived to corrected age of 36 wk were under observation. BPD, defined as oxygen dependency at $36 \mathrm{wk}$ adjusted gestational age, has developed in 25 of them (BPD group). 75 infants without BPD were included into the control group. Perinatal data, delivery room interventions, nasal CPAP as well as mechanical ventilation use and duration were compared in the groups.

Results: Infants from the BPD group were intubated in delivery room and needed initial mechanical ventilation more often than newborns from the control group (15 [60\%] vs. 27 [36\%], p< 0,05 and 22 [88\%] vs. 40 [53\%], $p<0,05$; accordingly). Duration of the initial period of mechanical ventilation was also significantly higher in infants from the BPD group (118 [69-162] hrs vs. 48 [24-86] hrs; $p<0,001)$. At the same time, newborns initially treated with CPAP less likely developed BPD (35 [47\%] in the control group as compared to 3 [12\%] in the BPD group; $p<0,01)$. This protective effect remained significant after controlling for gestational age, low 5 min Apgar score and severity of RDS (adjusted OR 0,46; $95 \%$ Cl 0,23-0,78).

Conclusions: Our data support the current concept of protective role of early noninvasive respiratory support for BPD development.

\section{PROTECTING THE VULNERABLE: A STUDY OF GONADAL EXPOSURE IN NEONATAL ABDOMINAL RADIOGRAPHS}

\author{
S.K. Sanka ${ }^{1}$, M. Bosemani², J. Hughes ${ }^{2}$, \\ A.E. Curley ${ }^{1}$
}

${ }^{1}$ Neonatal Intensive Care Unit, ${ }^{2}$ Paediatric Radiology Department, Addenbrookes Hospital NHS Trust, Cambridge, UK

Background: Neonates in intensive care often have multiple radiographs performed. Neonatal gonads would be expected to have high tissue radio-sensitivity and should be excluded from radiographs whenever possible. We aimed to assess in our unit how often gonads are present on neonatal abdominal radiographs.

Method: Retrospective audit of neonatal abdominal radiographs (July-October 2008).

Results: 50 radiographs were screened in 32 preterm and 18 term infants. The gonads were excluded (coned off appropriately) in 18/50 (36\%) of infants. This group had a median corrected gestational age of 33 (range 25-42) weeks. 64\%of abdominal radiographs had the gonads included in the radiation field. This group were of a similar median gestational $32(24-42)$ weeks. $74 \%$ of abdominal films were carried out in the first week of life.

Conclusion: Although gonadal shielding is not routinely performed in the UK appropriate coning in accordance with the lonising Radiations (Protection of Persons Undergoing Medical Examinations or Treatment) Regulations 1988 should be adhered to whenever practically possible. We demonstrated that two thirds of neonatal radiographs assessed did not achieve this. This was not related to gestational age or size. Although gonadal shields raise the issue of infection control, cost and masking of important nearby anatomy, repeated radiographic exposure in this vulnerable population of infants justifies re evaluation of practice in this area. 